\title{
Image Encryption Based on Permutation and Substitution Using Clifford Chaotic System and Logistic Map
}

\author{
Behrouz Fathi-Vajargah $^{1 *}$, Mohadeseh Kanafchian², Vassil Alexandrov ${ }^{3}$ \\ 1 Department of Statistics, University of Guilan, Rasht, Iran. \\ 2 Department of Mathematics, University of Guilan, Rasht, Iran. \\ ${ }^{3}$ ICREA, Barcelona Supercomputing Centre, Spain, Tecnologico de Monterrey, Mexico. \\ * Corresponding author. Email: fathi@guilan.ac.ir \\ Manuscript submitted March 21, 2017; accepted June 25, 2017. \\ doi: 10.17706/jcp.13.3.309-326
}

\begin{abstract}
Chaos theory is a field of study in mathematics that studies the behavior of dynamical systems that are highly sensitive to initial conditions. Many algorithms have been proposed for image encryption, but the chaotic encryption methods have a good combination of speed and high security. The key space for a good encryption scheme should be big enough to resist brute-force attacks. In this paper, first we investigate Clifford chaotic system for image encryption. In order to optimize the encryption system we increase the space key by using logistic map. The security and performance of the proposed method are analyzed by using the key space, entropy, statistical, differential and performance analysis. It can be concluded that the proposed image encryption system is suitable choice for practical applications.
\end{abstract}

Key words: Image encryption, chaos, clifford chaotic system, logistic map.

\section{Introduction}

Chaotic maps have been used in data encryption recently, because chaos is sensitive to initial conditions and control parameters, pseudo randomness and ergodicity [1], [2]. In the late 1980s, Matthews firstly used chaos to encrypt information and produced the key stream based on logistic map [3].

Habutsu et al proposed the first chaotic block cipher algorithm in 1991 [4]. Baptista [5] published a paper about chaotic encryption algorithm in 1998. Some of the 1D chaotic maps have been used in data encryption. 2D or higher dimensional chaotic maps are usually employed for image encryption.

In 1998, Friedrich has suggested that an image encryption system need to be repeated in two steps: diffusion and confusion [6], [7]. The chaos-based encryption schemes are composed of two processes generally: chaotic confusion of pixel positions by permutation process and diffusion of pixel grey values by a diffusion process. The pixels of plain image permute with a 2D chaotic map, while the latter changes the pixel values of each pixel one by one so that a small change for one pixel can spread out to almost all pixels in the whole image.

In 2004, Chen et al and Mao et al employed 3D chaotic Cat map and Baker map in permutation step of image encryption [8], [9].

Guam et al used a 2D Cat map and Chen's chaotic system for pixel position permutation and pixel value masking, in 2005 respectively [1]. Giesl [10] proposed an image encryption system using strange attractor in 2009. Today, because of the properties of chaotic systems, such as sensitivity to initial conditions and control parameters, pseudo randomness and ergodicity, chaos becomes popular in image encryption (Lian et al 
(2005) [11], Patidar et al (2010) [12], Wang and Lie (2010)[13], Wang et al (2012) [14], Wang and Guo (2014)[15], Hua et al. (2015) [16]).

Therefore, in order to improve the image encryption in [10] we proposed a new algorithm for image encryption using Clifford attractor and logistic map. This algorithm consists of two parts: the logistic map shuffle of the pixel position $\left(x_{0}, y_{0}\right)$ and the pixel value $z_{0}=P\left(x_{0}, y_{0}\right)$. We use $k_{2}$ times for shuffling the pixel position and value. Then we generate $\left(x_{k}, y_{k}\right)$ and $z_{k}$.

The rest of the paper is arranged as follows: section 2 describes the 1D logistic map. Section 3 constructs a new image encryption algorithm based on Clifford attractor and logistic map. The experimental results, analysis and comparison of the proposed image encryption system are shown in section 4, and finally section 5 concludes the paper.

\section{Logistic Map}

It is now widely understood that complex behavior in nature may have a simple underlying cause. Several examples of low-dimensional nonlinear maps have been extensively studied [5], [17], [18], [19]. On the other hand, many familiar processes are known to have regular periodic solutions. One of the most studied examples of a one-dimensional system capable including chaos is the logistic map [2], [20]

$$
x_{n+1}=r x_{n}\left(1-x_{n}\right)
$$

where $r$ is the control parameter.

Control parameter $r$ has a crucial role in behavior of the map and we can recognize the qualitative changes in the dynamics of map by varying the value $r$.

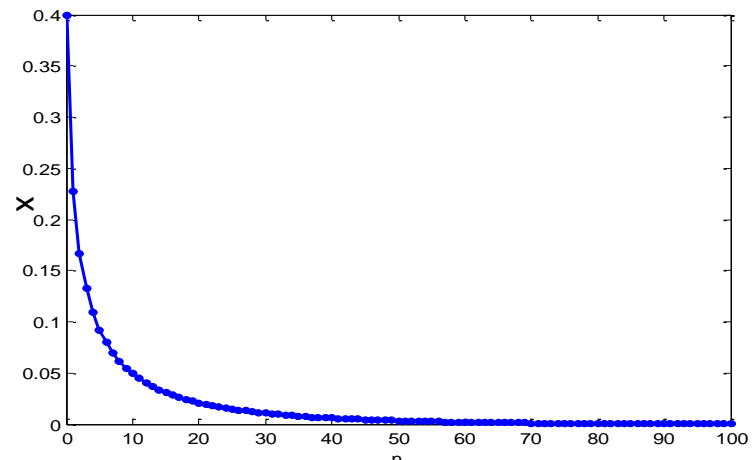

(a)

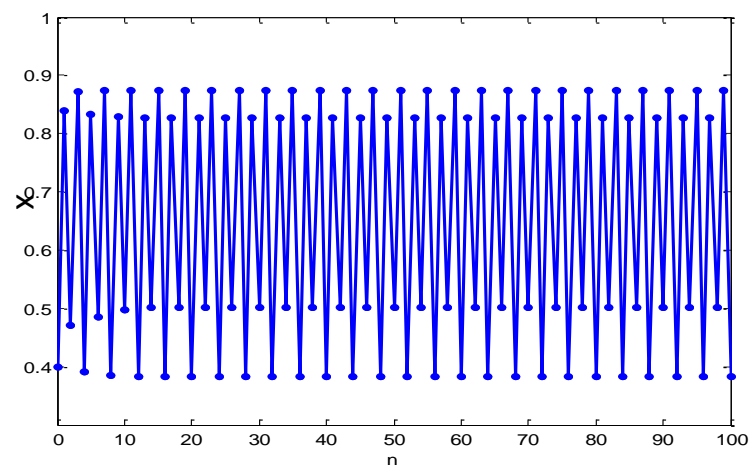

(c)

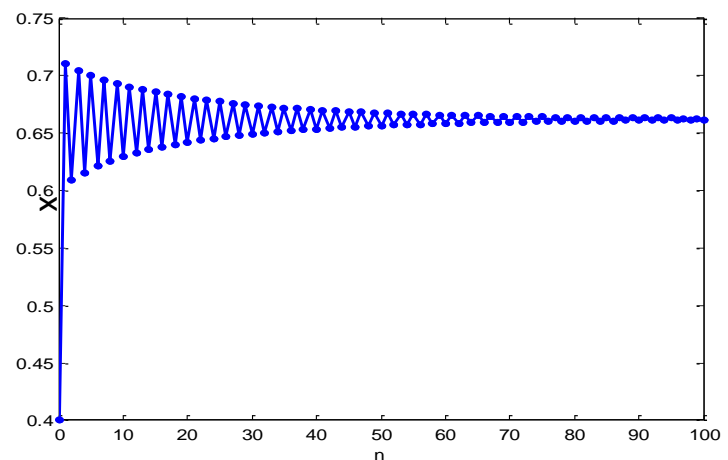

(b)

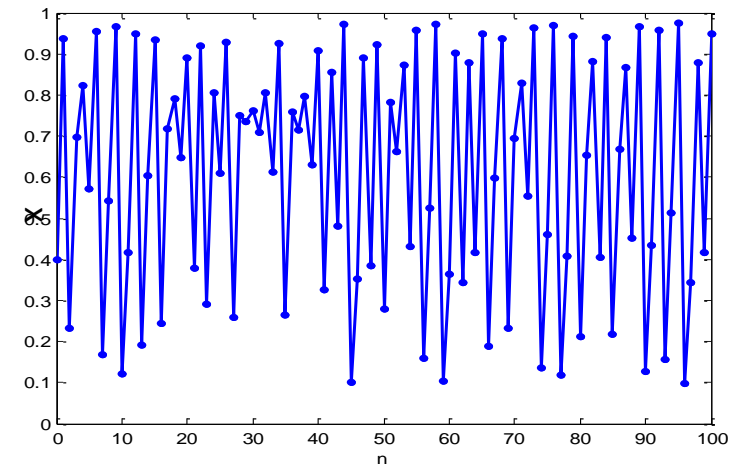

(d)

Fig. 1. First 100 points of logistic map with $x_{0}=0.4$ and different values of $r$ (a) $r=0.95$, (b) $r=2.95$, (c)

$$
r=3.5 \text {, (d) } r=3.9 \text {. }
$$


Upon repeated iteration, the solution will be one of the following cases depending upon the initial condition and its value: (1) it will converge to a stable fixed point (a point attractor), (2) it will converge to a periodic series of distinct values (a limit cycle), (3) it will yield a non periodic series of values within some bounded range of X (a chaotic strange attractor), or (4) it will diverge (attract to infinity).
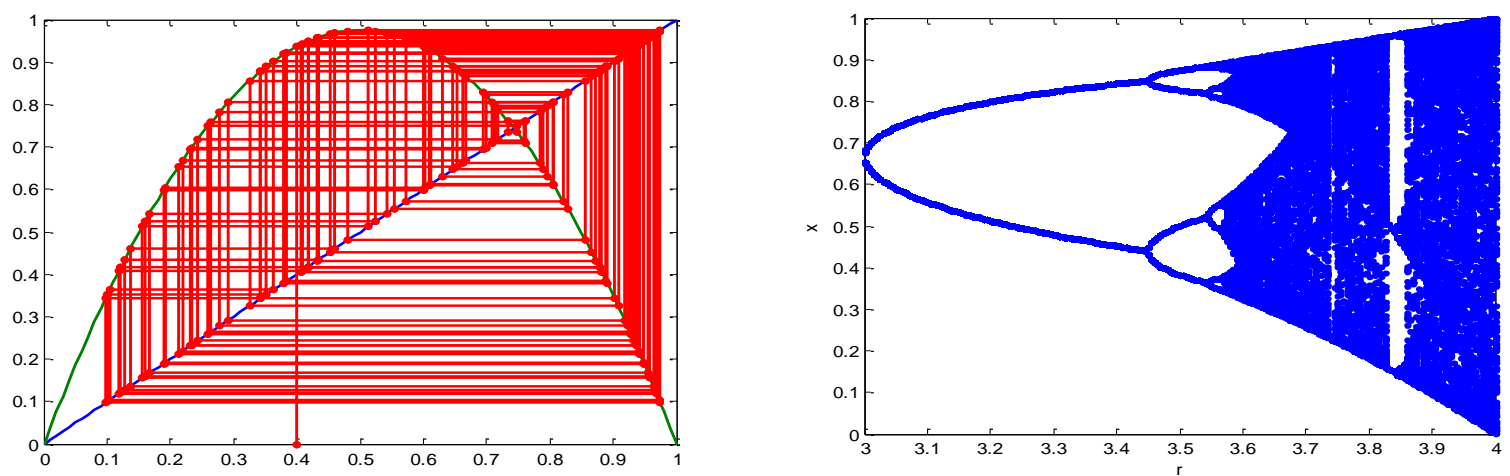

Fig. 2. (a) Behavior of logistic map with $x_{0}=0.4$ and $r=0.95$, (b) The bifurcation diagram for logistic map.

A feature of a chaotic system is sensitivity to initial conditions. If two trajectories which start off close to each other deviate more and more within creasing time, the system is said to be chaotic. The rate at which nearby trajectories deviate from each other with time is characterized by a quantity called the Lyapunov exponent. The Lyapunov exponent for the discrete chaotic system can be described as below

$$
h=\lim _{n \rightarrow \infty} \frac{1}{n} \ln \left|f^{\prime}(x)\right|
$$

where the parameter $n$ is the length of the sequence and $f$ is the chaos map. If $h>0$, it means that the chaotic system is currently chaotic with the certain condition. If the chaotic system is not chaotic under current condition.

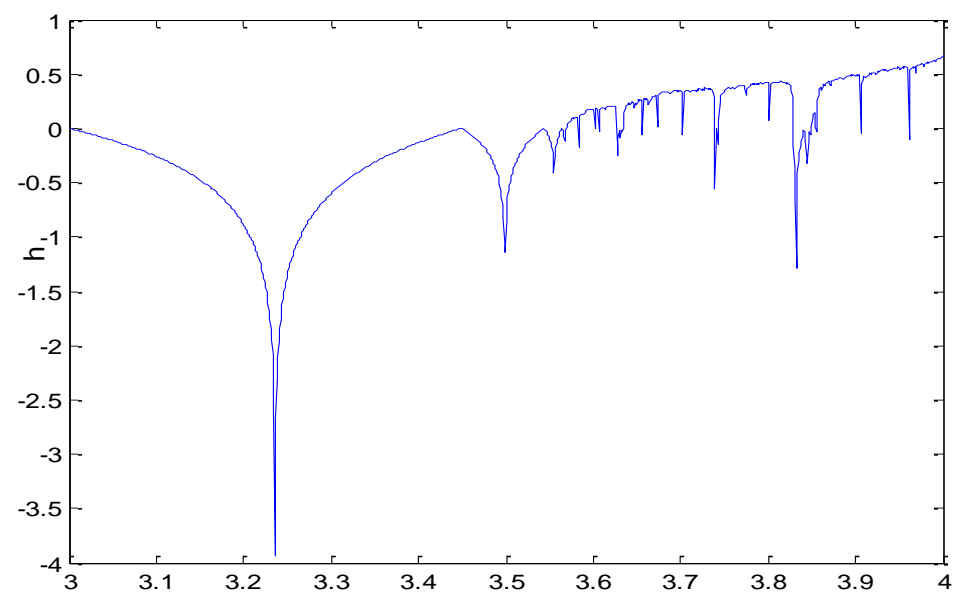

Fig. 3. Lyapunov exponent for logistic map.

For this case, the stable solutions are all in the range of $3.5 \leq r \leq 4$, and it is only within this range that the logistic equation represents a candidate model for a real physical process [21].

\section{Methodology}


Strange attractors [2], [10], [22]-[25] are complicated sets with a fractal structure to which chaotic dynamical systems evolve after enough long time. These attractors can be generated in several ways.

In general, a map is said to be dissipative if it does not preserve phase space volumes on each iterate. Therefore, the bounded region on the phase place will certainly shrink for the reason that map can be iterated and this is associated with the presence of an attractor within that region.

The proposed image encryption is a modification of the one suggested by Giesl in [10]. The algorithm in [10] does not create any encryption key, but uses attractor map for pixel permutation and diffusion directly. Parameters of attractor map play the role of encryption keys here and key space is very large due to their non-integer character. Chaos-based encryption has been done by means of so called Clifford system (attractor) [20],

$$
\begin{aligned}
& x_{n+1}=\sin \left(a y_{n}\right)+c \cos \left(a x_{n}\right) \\
& y_{n+1}=\sin \left(b x_{n}\right)+d \cos \left(b y_{n}\right) \\
& x_{n+1}=\sin \left(a y_{n}\right)+c \cos \left(a x_{n}\right) \\
& y_{n+1}=\sin \left(b x_{n}\right)+d \cos \left(b y_{n}\right) \\
& z_{n+1}=\sin \left(e y_{n}\right)+f \cos \left(e z_{n}\right)
\end{aligned}
$$

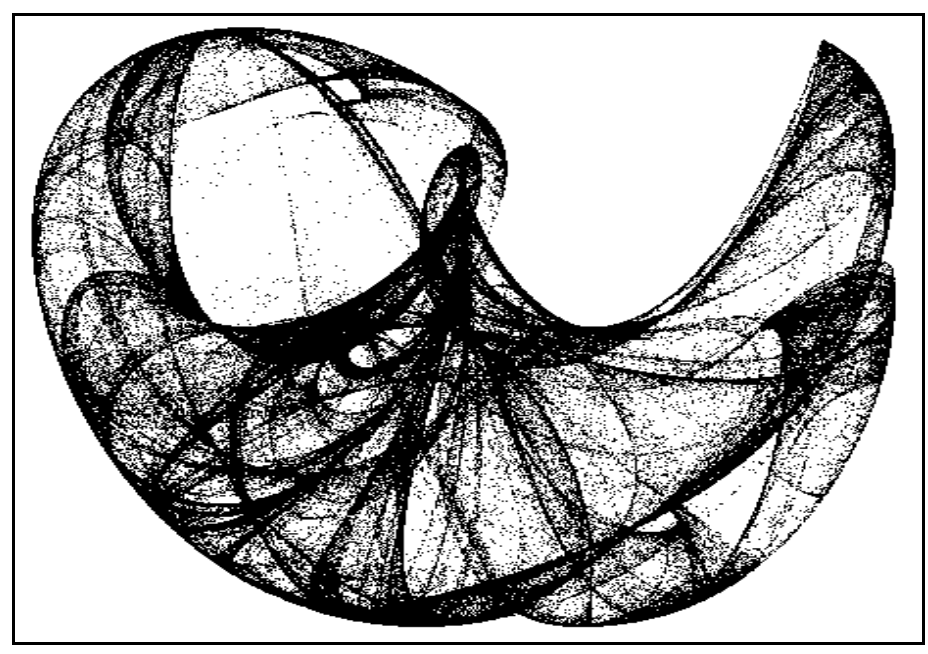

Fig. 4. Clifford attractor based on (4).

Implementation of image encryption is in two steps: the first step is image scrambling that the position of the pixels in the image is changed and the second step is pixel substitution. An image can be described by the position and the pixel value. This method can change the position and value of the image pixel and it converts the image from a plain digital image into a noise-polluted image.

Pixel substitution is implemented by XOR or other operation with other sequence or matrix to change plain image pixel value. The main parameters of the Clifford attractor are keys for the process of encryption. Pixel of image is used as the initial value of Clifford system. Equation in (4) is 3D extension which can be used for encryption of coordinates (pixel position) $\left(x_{0}, y_{0}\right)$ and the value of each pixel $P\left(x_{0}, y_{0}\right)$. New positions and modification value are gained after iterations and quantization. These positions are then used for pixel permutation and the modification value is XOR operated with original pixel value and the value of the previous pixel and encrypted pixel is gained this way [10]. According to Fig. 5, the previous algorithm was changed as follows: 


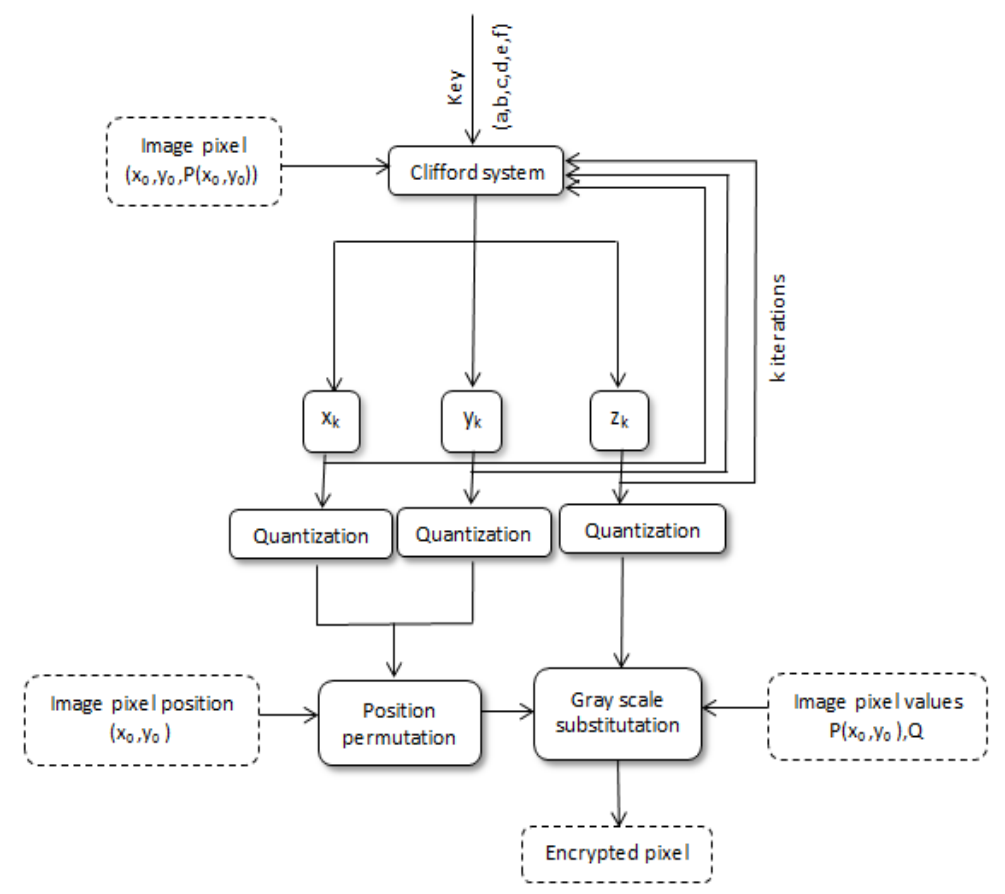

Fig. 5. Flowchart of encryption process suggested in [10].

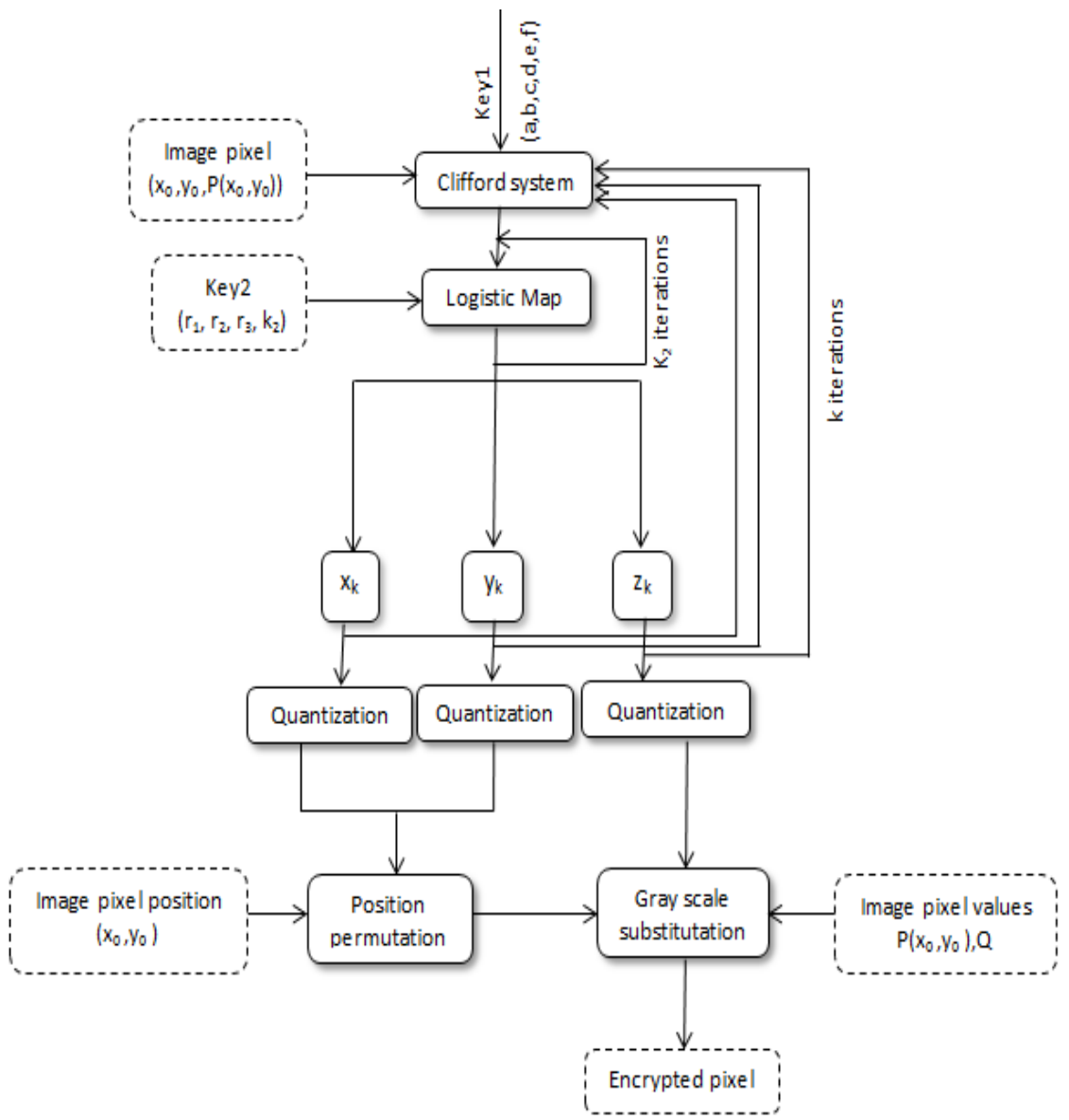

Fig. 6. Flowchart of proposed encryption process.

In this paper, encryption with Clifford chaotic system and the proposed Clifford chaotic system have been tested. The tested "message" for encryption is picture. We use the trigonometric strange attractor which is 
described by (4). In the new algorithm, first coordinates $\left(x_{0}, y_{0}\right)$ and value $P\left(x_{0}, y_{0}\right)$ of pixels put into a Clifford system. Next Logistic map iterate $k_{2}$ times with $r_{1}, r_{2}, r_{3}$ parameters. After quantization step, positions and modification value are gained. Encryption system contains 7 steps as follows:

$$
P\left(x_{0}, y_{0}\right) \leftrightarrow P\left(x_{k}, y_{k}\right) \oplus z_{k} \oplus Q
$$

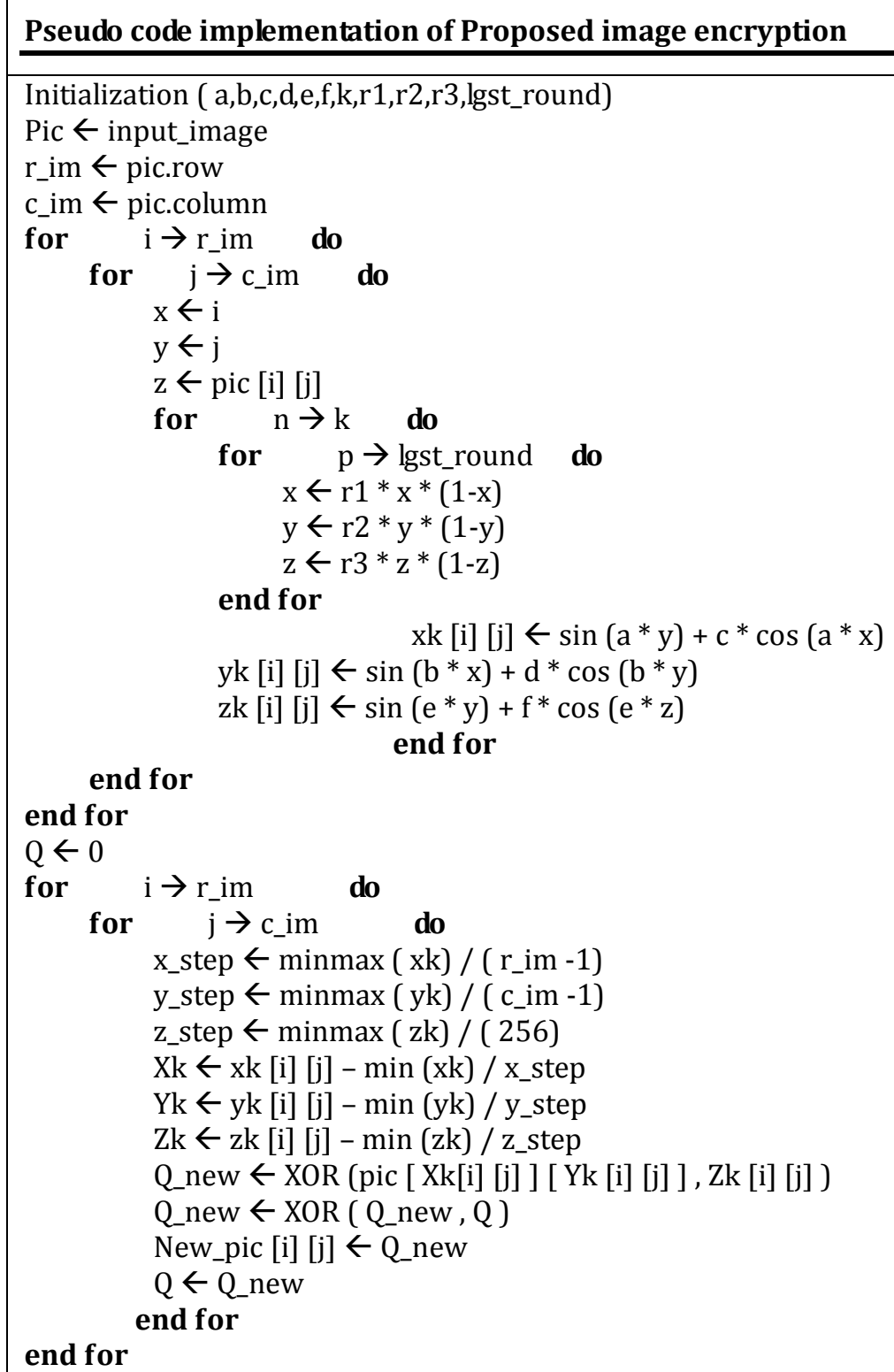

Step1. Key generation: Select parameters a, b, c, d, e, f of Clifford attractor and $r_{1}, r_{2}, r_{3}, k_{2}$ of Logistic map.

Step2. Image conversion matrix: Converts the tested image to a matrix. For grayscale images, the dimension of matrix is. $n$ is the height of the image while $m$ is the width. A color image can be converted into grayscale image.

Step3. Initial selection: as the initial process of encryption, select a pixel position $\left(x_{0}, y_{0}\right)$ and a pixel value $z_{0}=P\left(x_{0}, y_{0}\right)$.

Step4. New Clifford system: perform Logistic map and Clifford stranger to disorder the position and value pixel of image. Put $x_{0}, y_{0}$ and $z_{0}=P\left(x_{0}, y_{0}\right)$ into the Logistic map with $k_{2}$ iterations. 


$$
\begin{array}{ll}
x_{0}=r_{1} & x_{0}\left(1-x_{0}\right) \\
y_{0}=r_{1} & y_{0}\left(1-y_{0}\right) \\
z_{0}=r_{1} & z_{0}\left(1-z_{0}\right)
\end{array}
$$

Then Clifford attractor is used to shuffle pixel position and pixel value. The obtained $x_{0}, y_{0}, z_{0}$ are the initial values for Clifford equation.

$$
\begin{aligned}
& x_{k}=\sin \left(a y_{0}\right)+c \cos \left(a x_{0}\right) \\
& y_{k}=\sin \left(b x_{0}\right)+d \cos \left(b y_{0}\right) \\
& z_{k}=\sin \left(e y_{0}\right)+f \cos \left(e z_{0}\right)
\end{aligned}
$$

Set $x_{0}=x_{k}, y_{0}=y_{k}, z_{0}=z_{k}$ and iterate the above equation $k$ times. The original pixel at coordinates $\left(x_{0}, y_{0}\right)$ is swapped with the pixel at coordinates.

Step5. Reshaping matrices: Convert 2D matrices $x_{k}, y_{k}, z_{k}$ into 1D matrices.

Step6. Quantization: For the obtained vectors in step 5, determine minimum and maximum value of vectors. Then the range is divided between the minimum and maximum.

Step7. Changing values of pixels: Pixel value at coordinates $\left(x_{k}, y_{k}\right)$ is modified by XOR operation of value $z_{k}$ and then XOR operated with the value of the previous processed pixel Q. This process must be done for every pixel in the image.

\section{Simulation Results and Statistical Analysis}

The following tests are realized by MATLAB software on Intel Core (TM) i7, 2.2 GHz PC.

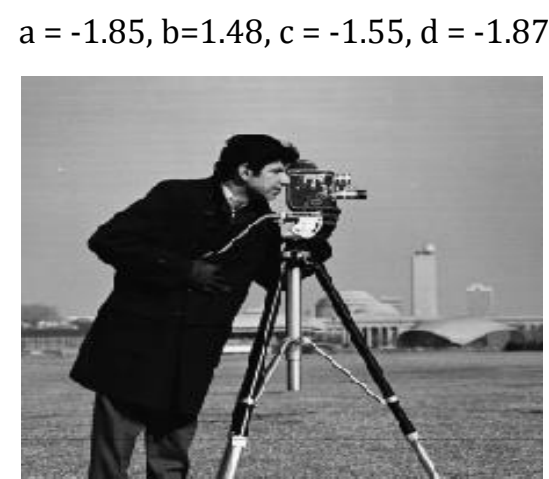

(a)

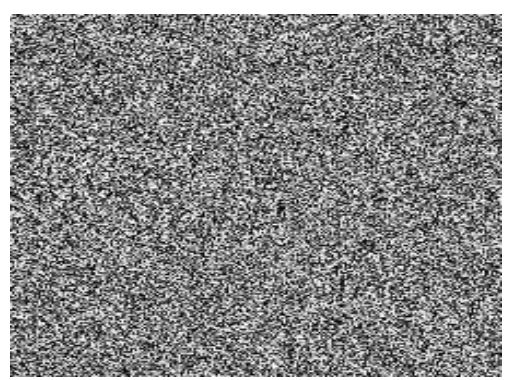

(c)

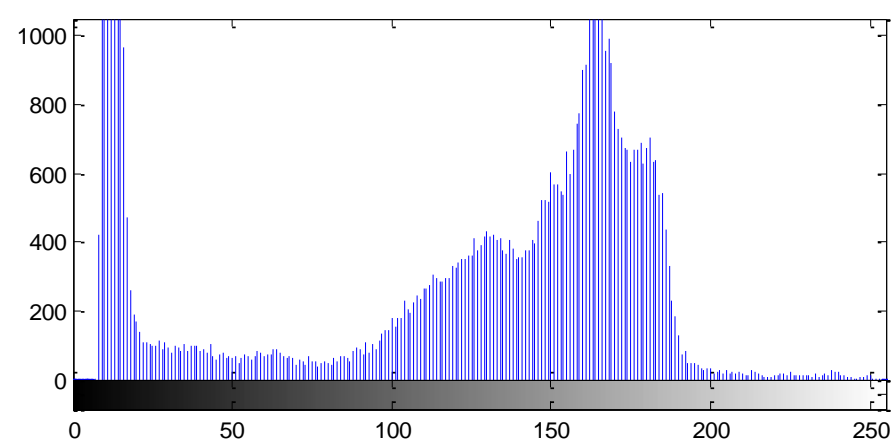

(b)

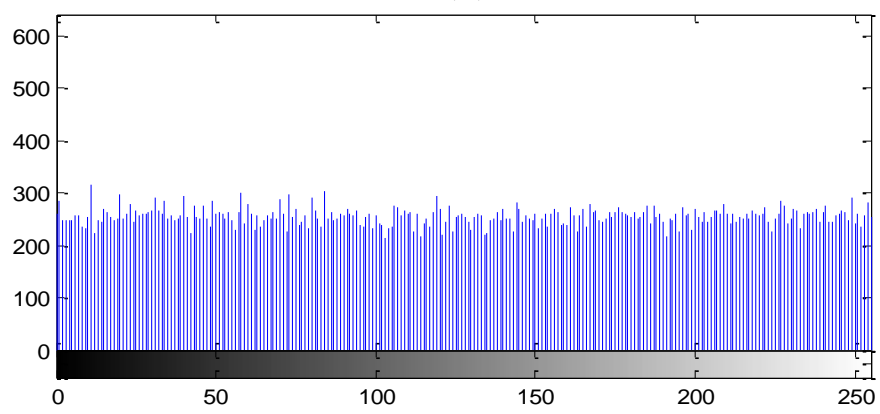

(d)

Fig. 7. (a) Plain image, (b) Histogram of plain image, (c) Cameraman image by proposed encryption system,

(d) Histogram of Cameraman image by proposed encryption system. 


\subsection{Key Space Analysis}

A good encryption algorithm should have large key space to prevent brute-force attacks which is defined to exhaust all the possible keys until the correct one. In the proposed method we increase the space key by using logistic map. So the number of parameters in the Clifford system increases from six to nine parameters. Therefore the size of the key space for the proposed system is larger than Clifford system.

\subsection{Encryption and Histogram Test}

Some experimental results are given in this section to demonstrate the efficiency of our scheme. The plain images are "Cameraman" and "Lena" with the size $256 \times 256$. Their cipher images are shown in Fig. 4(c) and Fig. 5(c). The histogram of the cipher images is shown in in Fig. 4 (d) and Fig. 5(d). As we can see, the pixel distribution of the cipher images is fairly uniform, which can greatly reduce the correlation between the pixel values. Both kinds of pictures show that the picture is really well encrypted.

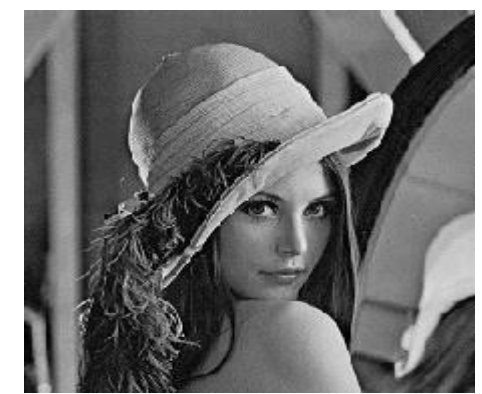

(a)

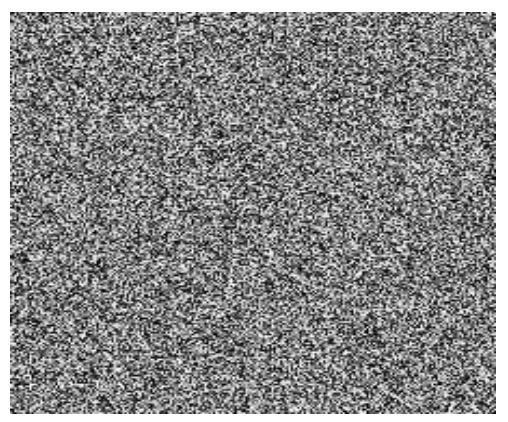

(c)

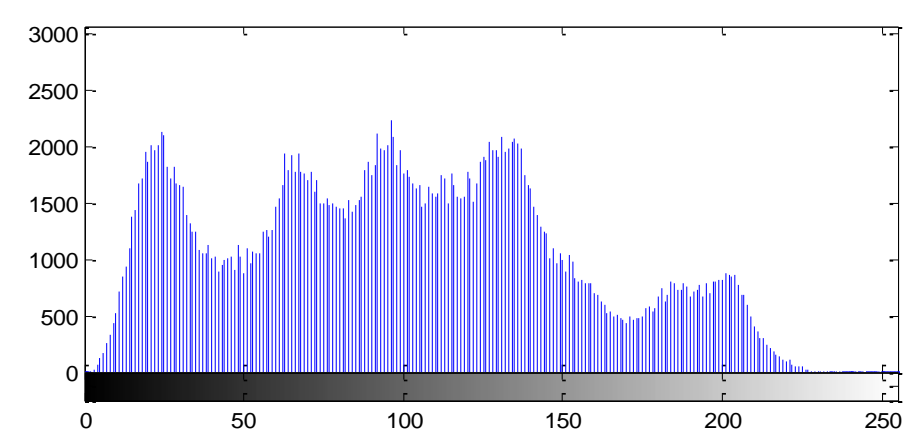

(b)

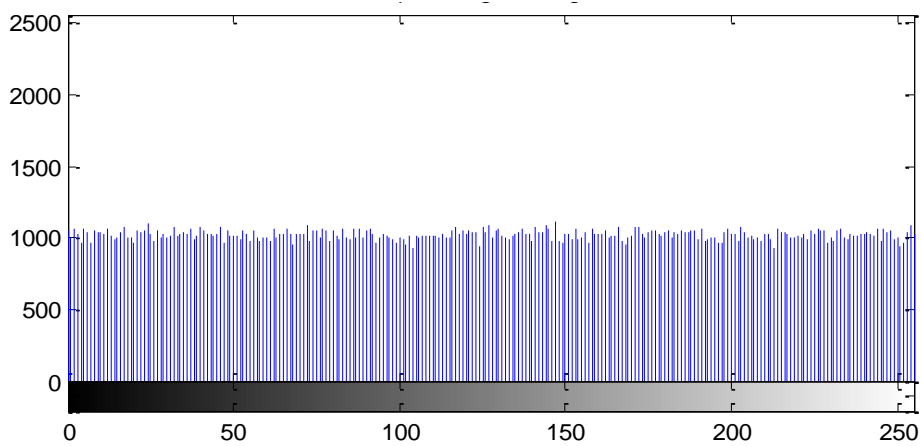

(d)

Fig. 8. (a) Plain image, (b) Histogram of plain image, (c) Lena image by proposed encryption system, (d) Histogram of Lena image by proposed encryption system.

\subsection{Chi-square Test}

The uniformity is justified by chi-square test, which is described by the following expression:

$$
\chi^{2}=\sum_{k=1}^{256} \frac{\left(O_{k}-E_{k}\right)^{2}}{E_{k}}
$$

$O_{k}=$ the observed frequencies of each gray level (0-255)

$E_{k}=$ the expected frequencies of each gray level (0-255) 
The following hypothesis is tested.

$$
\begin{gathered}
H_{0} \text { : The histogram of encrypted image has uniform distribution. } \\
H_{1} \text { : The histogram of encrypted image has not uniform distribution. }
\end{gathered}
$$

Assume significant level of $0.05, \chi^{2}(255,0.05)=291$. If $\chi_{\text {test }}^{2}<\chi^{2}(255,0.05)$ then the null hypothesis is not rejected and the distribution of the encrypted histogram is uniform. Following table shows chi-square values of different cipher images.

Table 1. Chi-square Test for Cipher Images

\begin{tabular}{lccc}
\hline \multirow{2}{*}{ image } & size & \multicolumn{3}{c}{ Chi-square } & \\
\cline { 3 - 4 } & & $\chi_{\text {test }}^{2}$ & $H_{0}$ \\
\hline Monalisa & $128 \times 128$ & 228.375000 & Accepted \\
Elaine & $225 \times 225$ & 221.597754 & Accepted \\
Lena & $256 \times 256$ & 262.940492 & Accepted \\
Cameraman & $256 \times 256$ & 250.085937 & Accepted \\
Puppy & $256 \times 256$ & 242.525729 & Accepted \\
Clock & $256 \times 256$ & 246.960937 & Accepted \\
Moon surface & $256 \times 256$ & 280.6328125 & Accepted \\
Boat & $512 \times 512$ & 252.0312500 & Accepted \\
Mandrill & $512 \times 512$ & 216.731558 & Accepted \\
Barbara & $512 \times 512$ & 211.583984 & Accepted \\
\hline
\end{tabular}

\subsection{Analysis for Correlation of Adjacent Pixels}

Correlation analysis of adjacent pixels is an important way to test the gray value relationship between adjacent pixels in cipher image. Adjacent pixels in plain image have high correlation and in ciphered image should have low correlation. We calculate the correlation coefficient of the plain and encrypted image ${ }^{r_{P, C}}$ by using the following formulas [26],

$$
\begin{gathered}
E(P)=\frac{1}{M . N} \sum_{i=1}^{M} \sum_{j=1}^{N} P(i, j), \quad E(C)=\frac{1}{M . N} \sum_{i=1}^{M} \sum_{j=1}^{N} C(i, j) \\
D(P)=\frac{1}{M \cdot N} \sum_{i=1}^{M} \sum_{j=1}^{N}[P(i, j)-E(P)]^{2} \\
\operatorname{cov}(P, C)=\frac{1}{M . N} \sum_{i=1}^{M} \sum_{j=1}^{N}[P(i, j)-E(P)][C(i, j)-E(C)] \\
r_{P, C}=\frac{\operatorname{cov}(P, C)}{\sqrt{D(P)} \sqrt{D(C)}}
\end{gathered}
$$

In these formulas $P(i, j)$ and $C(i, j)$ are gray values of the plain pixel and the encrypted one. $E(P)$, $E(C), D(P)$ and $D(C)$ are mathematical expectation of plain pixels $P(i, j)$, mathematical expectation of cipher pixels $C(i, j)$, variance of plain pixels $P(i, j)$ and variance of cipher pixels $C(i, j)$, respectively. In order to test the correlation of adjacent pixels in the cipher image which is encrypted according to the proposed scheme, a test is designed. 2000 pixels are chosen randomly from Lena image, and their correlation 
distribution of gray value is plotted.

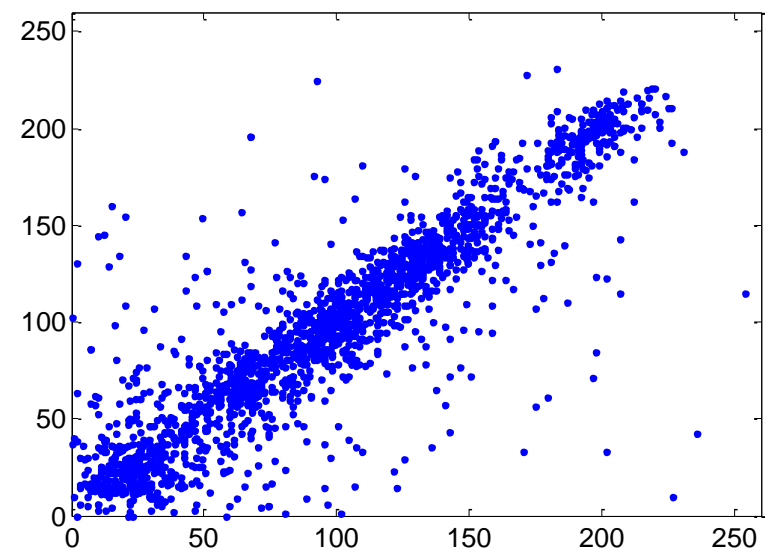

(a)

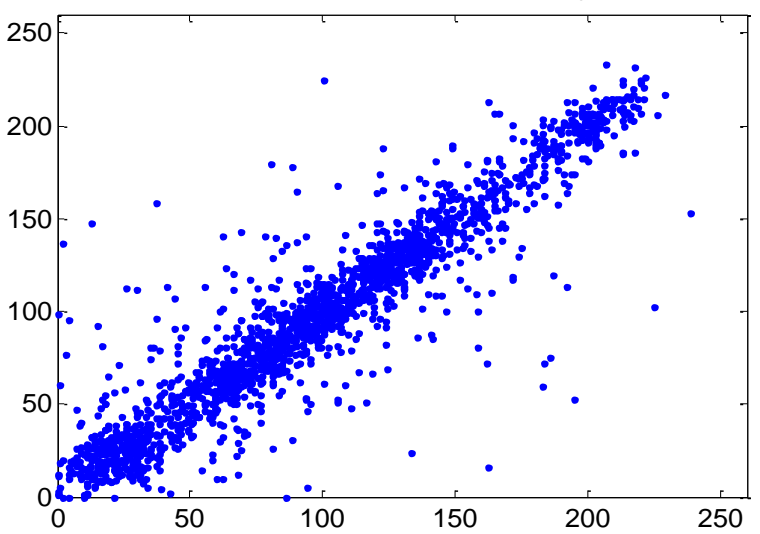

(c)

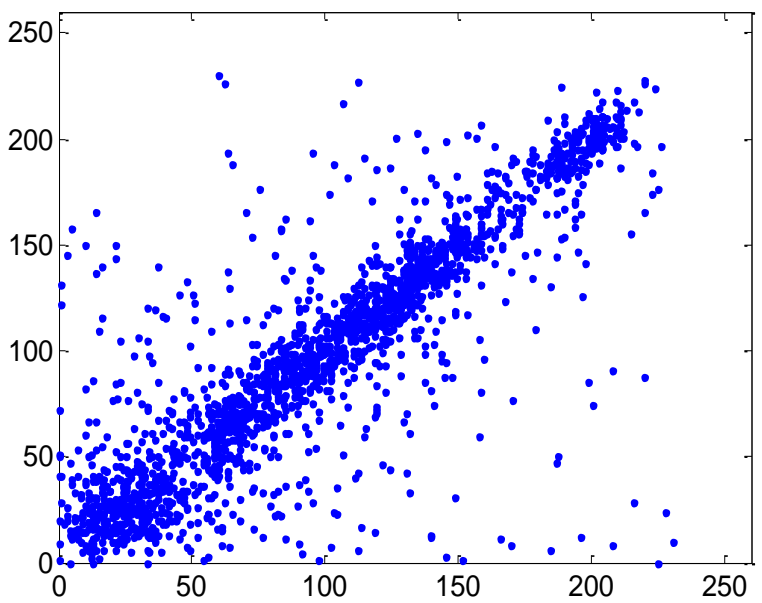

(e)

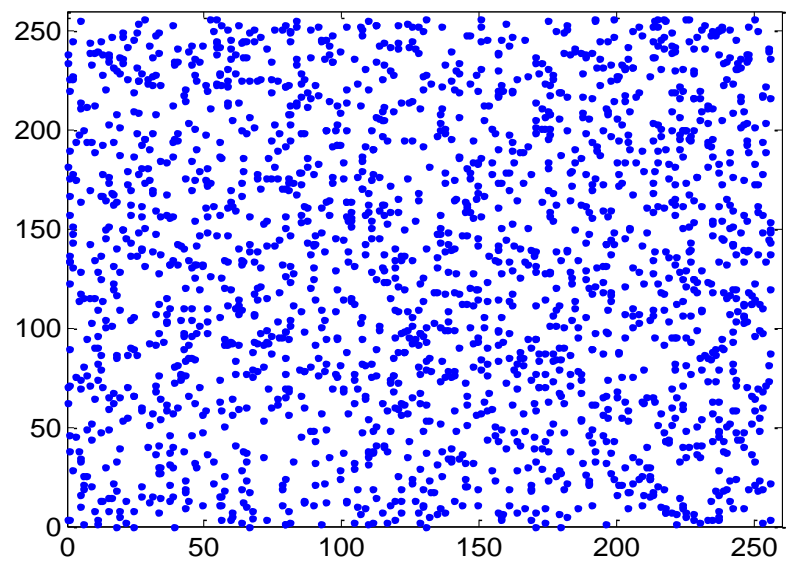

(b)
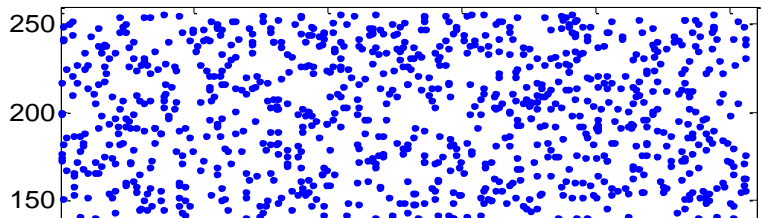

150

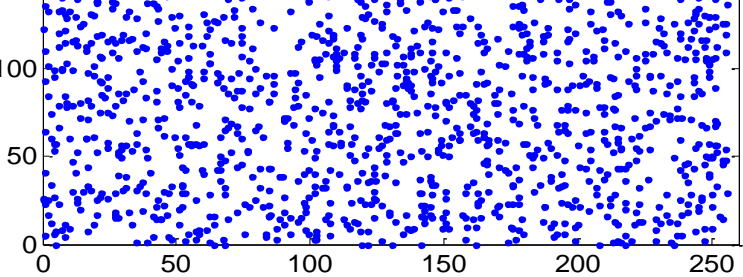

(d)

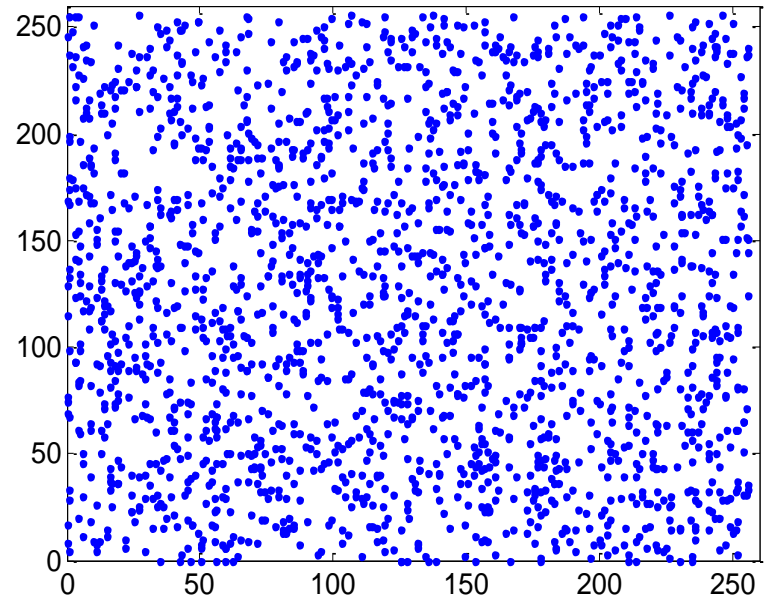

(f)

Fig. 9. Distribution of correlation coefficients: (a) distribution of the horizontal correlation coefficients of adjacent pixels of plain image, (b) distribution of the horizontal correlation coefficients of adjacent pixels of cipher image, (c) distribution of the vertical correlation coefficients of adjacent pixels of plain image, (d) distribution of the vertical correlation coefficients of adjacent pixels of cipher image, (e) distribution of the diagonal correlation coefficients of adjacent pixels of plain, (f) distribution of the diagonal correlation coefficients of adjacent pixels of cipher image.

The correlation coefficients of the adjacent pixels in horizontal, vertical and in diagonal are listed in Table 2. 
Table 2. Comparison of Correlation Coefficient of the Cipher Images in Three Algorithms

\begin{tabular}{cccc}
\hline \multicolumn{4}{c}{ Correlation } \\
\hline Method & Horizontal & Vertical & Diagonal \\
\hline Plain image & 0.891095 & 0.937048 & 0.873472 \\
Proposed method & -0.009264 & -0.000945 & -0.000284 \\
Clifford system & 0.029080 & -0.003002 & -0.000655 \\
Ref.[27] & 0.005336 & -0.002761 & 0.001662 \\
Ref.[13] & 0.02046 & 0.01748 & 0.002317 \\
Ref.[ 28] & 0.0368 & -0.0392 & 0.0068 \\
\hline
\end{tabular}

\subsection{Analysis for Correlation of Plain Image and Cipher Image}

We analyzed the correlation between various pairs of plain image and cipher image. Table 3 shows the results of correlation coefficient between the plain image and corresponding cipher image.

Table 3. Comparison of Correlation Coefficient of the Plain and Cipher Images by Using Two Methods

\begin{tabular}{lcc}
\hline \multirow{2}{*}{ image } & \multicolumn{2}{c}{ Correlation coefficient } \\
\cline { 2 - 3 } & Proposed method & Clifford system \\
\hline Lena & -0.000138 & 0.000809 \\
Cameraman & $-2.821731 \times 10^{-5}$ & 0.004672 \\
Puppy & $-6.908934 \times 10^{-5}$ & 0.002807 \\
Mona_lisa & 0.000700 & -0.007508 \\
Elaine & -0.002077 & -0.002793 \\
Moon surface & -0.004275 & 0.004224 \\
Clock & -0.000867 & 0.007334 \\
Boat & 0.001827 & 0.003237 \\
Mandrill & $-7.285256 \times 10^{-5}$ & 0.000911 \\
Barbara & -0.000140 & 0.001239 \\
\hline
\end{tabular}

The correlation coefficient value ranges from -1 to +1 . The correlation coefficients measured by using the proposed encryption system are very close to 0 . Therefore, the cipher and plain images are significantly different.

\subsection{Information Entropy Analysis}

Image information entropy can measure the distribution of image gray values. Image entropy is defined as [29],

$$
H=-\sum_{i=1}^{256} p_{i} \log p_{i}
$$

where $p_{i}$ represents the probability of occurrence of each pixel and log denotes the base 2 logarithm. The ideal value of the cipher information entropy is 8 . Table 4 and Table 5 demonstrate that the entropy of cipher images is very close to the ideal value by using the proposed method. 
Table 4. Comparison of Entropy Test for the Cipher Images

\begin{tabular}{cccc}
\hline \multirow{2}{*}{ Image } & \multicolumn{3}{c}{ Entropy } \\
\cline { 2 - 4 } & Plain image & Proposed method & Clifford system \\
\hline Lena & 7.651765 & 7.999233 & 7.998972 \\
Cameraman & 7.009716 & 7.997500 & 7.997452 \\
puppy & 7.453406 & 7.999414 & 7.999402 \\
Mona_lisa & 7.345221 & 7.997861 & 7.997836 \\
Clock & 6.705667 & 7.997284 & 7.997380 \\
Moon surface & 6.709312 & 7.996914 & 7.997010 \\
Boat & 7.191370 & 7.999385 & 7.999351 \\
Mandrill & 7.284855 & 7.999399 & 7.999411 \\
Elaine & 7.498110 & 7.998947 & 7.998888 \\
Barbara & 7.632119 & 7.999417 & 7.999282 \\
\hline
\end{tabular}

Table 5. Comparison of the Cipher Image Entropy Using Five Algorithms

\begin{tabular}{lccccc}
\hline Image & Proposed & Clifford system & Ref.[27] & Ref.[13] & Ref.[28] \\
\hline Lena & 7.999233 & 7.998972 & 7.997116 & 7.9990 & 7.9880 \\
\hline
\end{tabular}

Recently, the block entropy test was derived. In the following block entropy test [29], 100 non-overlapped blocks of the size $16 \times 16$ are randomly selected from each cipher image. The information entropy of each block is recorded via (10) and the average entropy is calculated.

Table 6. Entropy Test of Proposed Algorithm for Different Cipher Images

\begin{tabular}{ccccc}
\hline \multirow{2}{*}{ Image } & \multicolumn{4}{c}{ Entropy test } \\
\cline { 2 - 5 } & Global entropy & Actual block entropy & \multicolumn{2}{c}{ Theoretical block entropy } \\
\cline { 3 - 5 } & & & $\alpha=0.01$ & $\alpha=0.05$ \\
Lena & 7.999233 & 7.18390012 & Pass & Pass \\
Cameraman & 7.997500 & 7.17484195 & Pass & Pass \\
Puppy & 7.999414 & 7.17699590 & Pass & Pass \\
Mona_lisa & 7.997861 & 7.17077829 & Pass & Pass \\
Mandrill & 7.999399 & 7.17270896 & Pass & Pass \\
Clock & 7.997284 & 7.17073254 & Pass & Pass \\
Moon surface & 7.996914 & 7.17263831 & Pass & Pass \\
Boat & 7.999305 & 7.17555463 & Pass & Pass \\
Elaine & 7.998947 & 7.18004111 & Pass & Pass \\
Barbara & 7.999417 & 7.17968347 & Pass & Pass \\
\hline
\end{tabular}

\subsection{Sensitivity Analysis}

A good encryption algorithm should be sensitive to the plain image and the secret keys [30].

\subsubsection{Plain image sensitivity}

For calculation of NPCR and UACI, let us assume two cipher images $C_{1}$ and $C_{2}$ whose corresponding plain images have only one-pixel difference. NPCR and UACl are defined through the following formula as: 


$$
\begin{gathered}
N P C R=\frac{\sum_{i} \sum_{j} D(i, j)}{T} \times 100 \\
U A C I=\left[\sum_{i} \sum_{j} \frac{\left|C_{1}(i, j)-C_{2}(i, j)\right|}{L . T}\right] \times 100
\end{gathered}
$$

The symbols $T$ and $L$ denote the number of pixels in the cipher image and the largest allowed pixel intensity, respectively. $D(i, j)$ is defined as:

$$
D(i, j)= \begin{cases}0 & C_{1}(i, j)=C_{2}(i, j) \\ 1 & C_{1}(i, j) \neq C_{2}(i, j)\end{cases}
$$

Table 7 shows the real values of NCPR and UACI of cipher images by changing a pixel of the plain image.

Table 7.Comparison of NPCR and UACI of Cipher Images by Changing a Pixel of Plain Image

\begin{tabular}{cccc}
\hline Image & method & NPCR\% & UACI\% \\
\hline \multirow{3}{*}{ Lena } & Proposed & 99.626668 & 33.567139 \\
& Clifford system & 99.622414 & 33.472513 \\
& Ref.[16] & 99.60 & 99.66 \\
& Ref.[25] & 99.61 & 33.48 \\
\hline
\end{tabular}

\subsubsection{Key sensitivity}

A $128 \times 128$ gray image CT scan of brain is encrypted by using $\mathrm{a}=-1.85, \mathrm{~b}=1.48, \mathrm{c}=-1.55, \mathrm{~d}=-1.87$, $\mathrm{e}=$ $-4.32, \mathrm{f}=0.63, r_{1}=4, r_{2}=4, r_{3}=3.95$ as the first set of key. The key is changed slightly to be used to encrypt the same plain image. Slightly different kinds of key are listed below.

1. $\mathrm{a}=-1.8500001, \mathrm{~b}=1.48, \mathrm{c}=-1.55, \mathrm{~d}=-1.87, \mathrm{e}=-4.32, \mathrm{f}=0.63, r_{1}=4, r_{2}=4, r_{3}=3.95$

2. $\mathrm{a}=-1.85, \mathrm{~b}=1.4800001, \mathrm{c}=-1.55, \mathrm{~d}=-1.87, \mathrm{e}=-4.32, \mathrm{f}=0.63, r_{1}=4, r_{2}=4, r_{3}=3.95$

3. $\mathrm{a}=-1.85, \mathrm{~b}=1.48, \mathrm{c}=-1.5500001, \mathrm{~d}=-1.87, \mathrm{e}=-4.32, \mathrm{f}=0.63, r_{1}=4, r_{2}=4, r_{3}=3.95$

4. $\mathrm{a}=-1.85, \mathrm{~b}=1.48, \mathrm{c}=-1.55, \mathrm{~d}=-1.8700001, \mathrm{e}=-4.32, \mathrm{f}=0.63, r_{1}=4, r_{2}=4, r_{3}=3.95$

5. $\mathrm{a}=-1.85, \mathrm{~b}=1.48, \mathrm{c}=-1.55, \mathrm{~d}=-1.87, \mathrm{e}=-4.3199999, \mathrm{f}=0.63, r_{1}=4, r_{2}=4, r_{3}=3.95$

6. $\mathrm{a}=-1.85, \mathrm{~b}=1.48, \mathrm{c}=-1.55, \mathrm{~d}=-1.87, \mathrm{e}=-4.32, \mathrm{f}=0.6300001, r_{1}=4, r_{2}=4, r_{3}=3.95$

7. $\mathrm{a}=-1.85, \mathrm{~b}=1.48, \mathrm{c}=-1.55, \mathrm{~d}=-1.87, \mathrm{e}=-4.32, \mathrm{f}=0.63, r_{1}=4.0000001, r_{2}=4, r_{3}=3.95$

8. $\mathrm{a}=-1.85, \mathrm{~b}=1.48, \mathrm{c}=-1.55, \mathrm{~d}=-1.87, \mathrm{e}=-4.32, \mathrm{f}=0.63, r_{1}=4, r_{2}=3.9999999, r_{3}=3.95$

9. $\mathrm{a}=-1.85, \mathrm{~b}=1.48, \mathrm{c}=-1.55, \mathrm{~d}=-1.87, \mathrm{e}=-4.32, \mathrm{f}=0.63, r_{1}=4, r_{2}=4, r_{3}=3.9500001$

Table 8 shows the NPCR and UACI between the cipher images with the correct key and slightly different keys mentioned above. The cipher images with the correct and slightly different keys are compared pixel-by-pixel. This table shows that more than $99.60 \%$ pixels in the cipher image change their gray rate when one of the keys just changes $10^{-6}$. If a slightly modified key is used to decrypt the cipher image, the 
decryption fails completely.

Table 8. NPCR and UACI between Cipher Image with Key a $=-1.85, \mathrm{~b}=1.48, \mathrm{c}=-1.55, \mathrm{~d}=-1.87, \mathrm{e}=-4.32$, $\mathrm{f}$ $=0.63, r_{1}=4, \quad r_{2}=4, \quad r_{3}=3.95$ and Other Cipher Images with Slightly Different Keys

\begin{tabular}{clc}
\hline Kind of modified key & NPCR \% & UACI\% \\
\hline (1) & 99.664306 & 33.157169 \\
(2) & 99.627685 & 33.602558 \\
(3) & 99.603271 & 33.536951 \\
(4) & 99.609375 & 33.728769 \\
(5) & 99.664306 & 33.591356 \\
(6) & 99.676513 & 33.635636 \\
(7) & 99.609375 & 33.191253 \\
(8) & 99.633789 & 33.302504 \\
(9) & 99.688720 & 33.790785 \\
\hline
\end{tabular}

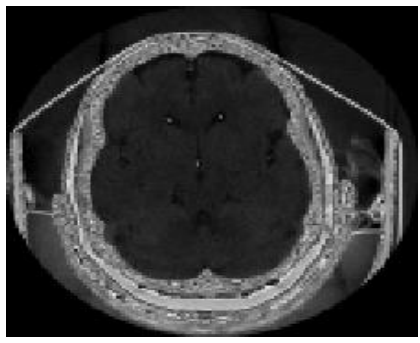

(a)

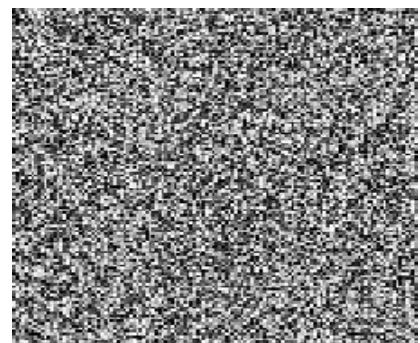

(b)

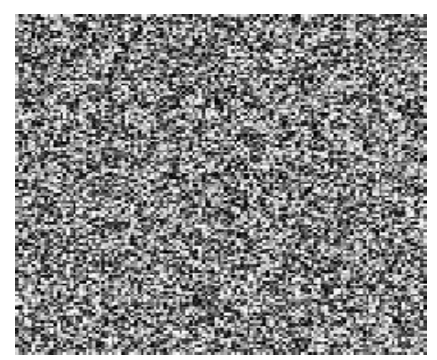

(c)

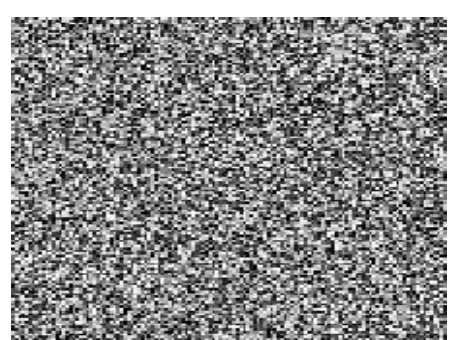

(d)

Fig. 10. Key sensitivity analysis for encryption process for CT scan of brain Image (a) Plain Image (b) Cipher image with correct key (c) Cipher image by changing parameter b, b=1.4800001 (d) Cipher image by

$$
\text { changing parameter } r_{3}=3.9500001 \text {. }
$$

Comparison of NPCR and UACI for five cipher images by using proposed and Clifford systems is listed in Table 9.

Table 9. Comparison of NPCR and UACI between Cipher Image with Key a $=-1.85, b=1.48, c=-1.55, d=-1.87$, $\mathrm{e}=-4.32, \mathrm{f}=0.63, r_{1}=4, r_{2}=4, r_{3}=3.95$ and Other Cipher Images by Changing Parameter $\mathrm{b}, \mathrm{b}=1.4800001$

\begin{tabular}{ccccc}
\hline & \multicolumn{3}{c}{ Proposed method } & Clifford system \\
\cline { 2 - 5 } Image & NPCR\% & UACI\% & NPCR \% & UACI\% \\
\hline Lena & 99.626668 & 33.567139 & 99.599711 & 33.402580 \\
Puppy & 99.611780 & 33.523781 & 99.623159 & 33.488289 \\
Mandrill & 99.620887 & 33.480585 & 99.606999 & 33.518280 \\
Elaine & 99.639835 & 33.498231 & 99.613497 & 33.452525 \\
Barbara & 99.613511 & 33.521040 & 99.607467 & 33.476234 \\
\hline
\end{tabular}

\subsubsection{Randomness test for NPCR and UACI}

Recently, the NPCR and UACI randomness tests [31] were derived, and thus these scores can be used for the qualitative test as well. The critical NPCR score $N_{\alpha}{ }^{*}$ associated with one-side hypothesis test under 
the ${ }^{\alpha}$ level of significance is shown in (14), where the $\Phi^{-1}($.$) is the inverse CDF of the standard Normal$ distribution $N(0,1)$. If an actual NPCR score is above $N_{\alpha}{ }^{*}$, then the null hypothesis that the difference of cipher images from the slightly different plain images are random-like. In other words, the NPCR test is passed if the actual NPCR score is greater than $N_{\alpha}{ }^{*}$.

$$
N_{\alpha}^{*}=\frac{L-\Phi^{-1}(\alpha) \sqrt{L / T}}{L+1}
$$

In addition, the critical UACI scores associated with the two-side hypothesis test under $\alpha$-level of significance are shown in (15). As a result, the UACI test is passed if the actual UACI score falls in the interval of $\left(U_{\alpha}^{*-}, U_{\alpha}^{*+}\right)$.

$$
\left\{\begin{array}{l}
U_{\alpha}^{*-}=\mu_{U}-\Phi^{-1}(\alpha / 2) \sigma_{U} \\
U_{\alpha}^{*+}=\mu_{U}+\Phi^{-1}(\alpha / 2) \sigma_{U}
\end{array}, \quad \mu_{U}=\frac{L+2}{3 L+3}, \quad \sigma_{U}^{2}=\frac{(L+2)\left(L^{2}+2 L+3\right)}{18(L+1)^{2} L T}\right.
$$

The results of NPCR and UACI scores from the proposed image encryption method are shown in Table 10.

\begin{tabular}{|c|c|c|c|c|c|c|}
\hline \multirow{3}{*}{$\begin{array}{l}\text { Image } \\
\text { name }\end{array}$} & \multicolumn{3}{|c|}{ NPCR scores\% } & \multicolumn{3}{|c|}{ UACI scores\% } \\
\hline & \multirow{2}{*}{$\begin{array}{l}\text { Actual } \\
\text { NPCR }\end{array}$} & \multicolumn{2}{|c|}{ Theoretical NPCR } & \multirow{2}{*}{$\begin{array}{c}\text { Actual } \\
\text { UACI }\end{array}$} & \multicolumn{2}{|c|}{ Theoretical UACI } \\
\hline & & $N_{0.01}^{*} 99.5810$ & $N_{0.05}^{*} 99.5893$ & & $\begin{array}{c}U_{0.01}^{*_{-}} / U_{0.01}^{*+} \\
33.3445 / 33.5826\end{array}$ & $\begin{array}{c}U_{0.05}^{*_{-}} / U_{0.05}^{*_{+}} \\
33.3730 / 33.5541\end{array}$ \\
\hline $\begin{array}{c}\text { Lena } \\
512 \times 512\end{array}$ & 99.803924 & Pass & Pass & 25.048828 & Fail & Fail \\
\hline $\begin{array}{c}\text { Barbara } \\
512 \times 512\end{array}$ & 99.803924 & Pass & Pass & 33.384517 & Pass & Pass \\
\hline $\begin{array}{c}\text { Boat } \\
512 \times 512\end{array}$ & 99.587991 & Pass & Fail & 33.412356 & Pass & Pass \\
\hline
\end{tabular}

Table 10. NPCR and UACI Tests for Cipher Images

\subsection{MSE, MAE and PSNR}

The difference between the plain image and cipher image can be measured with mean square error (MSE) and mean absolute error (MAE) criteria. MSE and MAE values are stated in (15) and (16), respectively.

$$
\begin{gathered}
M S E=\frac{\sum_{i} \sum_{j}(P(i, j)-C(i, j))^{2}}{T} \times 100 \\
M A E=\frac{\sum_{i} \sum_{j}|P(i, j)-C(i, j)|}{T} \times 100
\end{gathered}
$$


The symbol $T$ denotes the number of pixels in the cipher image. The larger value of MAE is better for the image security. Peak signal-to-noise ratio (PSNR) is the ratio between the plain image and the cipher image. The PSNR is defined as below:

$$
P S N R=10 \log _{10}\left(\frac{M A X_{I}^{2}}{M S E}\right)
$$

where $M A X_{I}$ is the maximum possible pixel value of the plain image. The higher PSNR, the closer the encrypted image is to the original. In general, a higher PSNR value should correlate to a higher quality image. In good encryption, scheme PSNR should be as low as possible. MSE, MAE and PSNR values of the cipher image are reported in Table 11.

Table 11. The Results of MSE, MAE and PSNR Tests

\begin{tabular}{crcr}
\hline Image & MSE & MAE & PSNR \\
\hline Clock & 12228.46 & 59.013153 & 7.257085 \\
Airplane & 10915.78 & 66.1103363 & 7.750255 \\
Moon surface & 6252.83 & 67.188827 & 10.170032 \\
Woman & 11973.73 & 89.400894 & 7.348506 \\
Peppers & 8313.78 & 75.225265 & 8.932815 \\
\hline
\end{tabular}

From Table 11, the results of MSE and MAE tests are high values which can ensure the resistance of the algorithm against any differential attacks.

\section{Conclusion}

The reported paper aimed at developing a secure algorithm for image encryption. The first step of implementation is image scrambling that the position of the pixels in the plain image is changed by using Logistic map and Clifford attractor. After quantization step, positions and modification value are gained.

A computer simulation is used to evaluate and compare the encrypted images by the proposed algorithm with other methods. The results of computer simulation show good results in the proposed method by using the nature of Chaos mechanism to achieve the improvements of the Clifford system. These comparisons were based on chi-square test of histogram, correlation coefficients of pixels, the number of pixel change rate (NPCR), entropy test, unified average changing intensity (UACI), mean square error (MSE), mean absolute error (MAE), peak signal-to-noise ratio (PSNR), key space, and sensitivity analysis.

In ideal condition, there should not be any relation between the pixels of the encrypted image. As shown in Fig. 9, Table 2 and Table 3, the absolute value of correlation coefficient of the proposed system is less than image encryption algorithms in [28], [10], [13] and [27]. Furthermore, the block entropy test also supports that the proposed method is good for image encryption. The results of statistical tests provided sufficient evidence for the superiority of the proposed chaotic image encryption system over other algorithms.

\section{References}

[1] Arrowsmith, D., \& Place, C. (1992). Dynamical Systems: Differential Equations, Maps, and Chaotic Behavior. New York: Chapman \& Hall.

[2] Kocarev, L., \& Lian, S. (2011). Chaos-Based Cryptography, Theory, Algorithms and Applications. New York: Springer. 
[3] Matthews, R. (1989). On the derivation of a chaotic encryption algorithm. Cryptology, 8, 29-41.

[4] Habutsu, T., Nishio, Y., Sasase, I., \& Mori, S. (1991). A secret key cryptosystem by iterating chaotic map, lect. notes comput. Sci Advances in Cryptology - EuroCrypt, 547, 127-140.

[5] Baptista, M. S. Cryptography with chaos. Physics Letters A, 240, 50-54.

[6] Fridrich, J. (1997). Image encryption based on chaotic maps. Proceedings of IEEE Conference on Systems, Man, and Cybernetics (pp. 1105-1110).

[7] Fridrich, J. (1998). Symmetric ciphers based on two-dimensional chaotic maps. Int. J. Bifurcation and Chaos, $8(6), 1259-1284$.

[8] Chen, G., Mao, Y. B., \& Chui, C. K. (2004). A symmetric image encryption scheme based on 3D chaotic cat maps. Int. J. Chaos, Solitons and Fractals, 21, 749-761.

[9] Mao, Y. B., Chen, G., \& Lian, S. G. (2004). A novel fast image encryption scheme based on the 3D chaotic baker map. Int J Bifurcat Chaos, accepted, 14(10), 3613-3624.

[10] Giesl, J., \& Vlcek, K. (2009). Image encryption based on strange attractors. ICGST-GVIP Journal, 9(2), 19-26.

[11] Lian, S. G., Sun, J., \& Wang, Z. (2005). A block cipher based on a suitable use of chaotic standard map, chaos. Soltians and Fractals, 26(1), 117-129.

[12] Patidar, V., Pareek, N. K., Purohit, G., \& Sud, K. (2010). Modified substitution-diffusion image cipher using chaotic standard and logistic maps. Commun Nonlinear Sci Number Simul, 15, 2894-2906.

[13] Wang, X., \& Lie, Y. (2012). A novel chaotic image encryption algorithm based on water wave motion and water drop diffusion models. Optics Communications, 282, 4033-4042.

[14] Wang, X. Y., Teng, L., \& Qin, X. (2012). A novel color image encryption algorithm based on chaos. Signal Process, 92, 1101-1108.

[15] Wang, X. Y., \& Gua, K. (2014). A new image alternate encryption algorithm based on chaotic map. Nonlinear Dyn, 67, 1943-1950.

[16] Hua, Z. Y., Zhou, Y. C., Pun, C. M., \& Chen, C. L. (2015). 2D Sine logistic modulation map for image encryption. Inf Sci, 297, 80-94.

[17] Henon, M. (1976). Commun. Math. Phys.

[18] Lorenz, E. N. (1963). Atmos. J. Sci. 20, 130.

[19] May, R. N. (1976). Nature, 261, 459.

[20] Zelinka, I., Celikovsky, S., Richter, H., \& Chen, G. (2010). Evolutionary Algorithms and Chaotic Systems. Berlin Heidelberg: Springer-Verlag.

[21] Sprott, J. C. (1993). How common is chaos? Physics Letters A, 173, 21-24.

[22] Pickover, C. (1988). A note on rendering 3-D strange attractors. Computers \& Graphics, 12, 263-267.

[23] Rossler, O. E. (1976). Phys. Lett. A, 57, 397.

[24] Sprott, J. C. (1993). Strange attractors: Creating patterns in chaos. M\&T Books.

[25] Yen, J. C. \& Guo, J. I. (2000). A new chaotic key-based design for image encryption and decryption. Proceedings of IEEE International Symposium on Circuits and Systems. (pp. 49-52).

[26] Gao, T. G., \& Chen, Z. Q. (2008). Image encryption based on a new total shuffling algorithm. Chaos Solitons Fractals, 38(1), 213-220.

[27] Yue, W., Yicong, Z., \& Joseph, P. (2014). Design of image cipher using Latin squares. Information Sciences, 264, 317-339.

[28] Fu, C., Lin, B., Miao, Y., Liu, X., \& Chen, J. (2011). A novel chaos-based bit-level permutation scheme for digital image encryption. Optics Commun, 284, 5415-5423.

[29] Wu, Y., Noonan, J. P., \& Agaian, S. (2011). Shannon entropy based randomness measurement and test for image encryption. Information Sciences, 1-23.

[30] Guan, Z. H., Huang, F. J., \& Guan, W. J. (2005). Chaos-based image encryption algorithm. Physics Letters A, 346, 153-157. 
[31] Wu, Y, Noonan, J. P, \& Agaian, S. (2011). NPCR and UACI randomness tests for image encryption. Multidisciplinary Journals in Science and Technology, Journal of Selected Areas in Telecommunications (JSAT), 31-38.

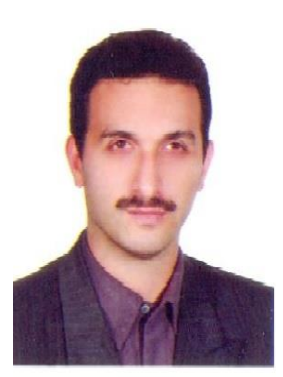

Behrouz Fathi vajargah has got the PhD degree from University of Reading, England in 2001 and now he is the associate professor in University of Guilan, Guilan, Iran. He has published more than 100 papers in international journals and conferences. Now, he is working on computations based on simulation and Monte Carlo methods.

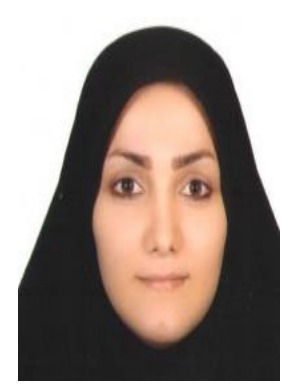

Mohadeseh Kanafchian is a Ph.D student in applied mathematics, stochastic processes field at University of Guilan.

She has got her M.Sc degree in applied mathematics, stochastic processes field from University of Guilan. Her research focus on Monte Carlo methods in cryptography.

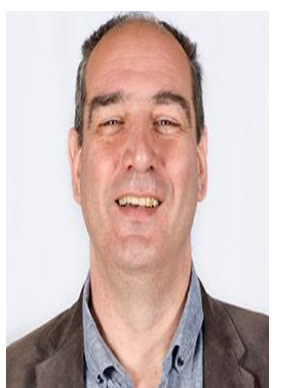

Vassil Alexandrov is an ICREA research professor in computational science at BSC since 2010. He obtained the MSc degree in applied mathematics from Moscow State University, Russia in 1984, and a PhD in parallel computing from Bulgarian Academy of Sciences in 1995. He has held previous positions at the University of Liverpool in UK, and Department of Statistics and Computational Mathematics and Computer Science from 1994 to 1999, the University of Reading in UK, School of Systems Engineering from 1999 to 2010. He was the professor of computational science leading the computational science research group until September 2010, and as the director of the centre for advanced computing and emerging technologies until July 2010. He has published over 120 papers in renowned refereed journals and international conferences in the area of his research expertise. 